\title{
LAPTM4B overexpression is an independent prognostic marker in ovarian carcinoma
}

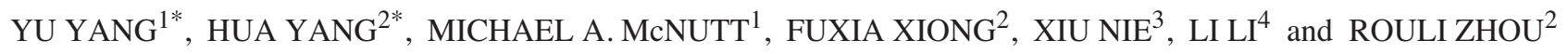 \\ Departments of ${ }^{1}$ Pathology and ${ }^{2}$ Cell Biology, Peking University Health Science Center, Beijing 100083, \\ ${ }^{3}$ Department of Pathology, Union Hospital Affiliated to Tongji Medical College, Huazhang \\ University of Science and Technology, Wuhan 430022, ${ }^{4}$ Department of Obstetrics and \\ Gynecology, Peking University Third Hospital, Beijing 100083, P.R. China
}

Received May 20, 2008; Accepted June 30, 2008

DOI: 10.3892/or_00000112

\begin{abstract}
Lysosome-associated protein transmembrane $4 \beta$ (LAPTM4B) is a member of the mammalian 4-tetratransmembrane spanning protein superfamily, which is strongly expressed in some solid malignancies. The present study investigated the correlation of LAPTM4B expression with clinicopathological features and prognosis in ovarian carcinoma. Expression of LAPTM4B was evaluated semiquantitatively by immunohistochemistry in 85 cases of ovarian carcinoma. High LAPTM4B expression was found in $54(63.5 \%)$ of these 85 carcinomas, and was positively correlated with FIGO stage, histological subtype and residual tumor after primary surgery, but not with age and tumor grade. Patients with high LAPTM4B expression had significantly poorer overall survival (OS) and progressionfree survival (PFS) $(\mathrm{P}<0.001$ and $\mathrm{P}<0.001$, respectively) compared to patients with low expression of LAPTM4B. On multivariate analysis, LAPTM4B expression was found to be an independent prognostic factor for OS and PFS $(\mathrm{P}=0.01$ and $\mathrm{P}=0.03$, respectively). These results showed that high LAPTM4B expression was associated with progression of ovarian carcinoma and correlated with unfavorable clinical outcome, suggesting that LAPTM4B may be a clinically useful prognostic indicator for ovarian carcinoma.
\end{abstract}

\section{Introduction}

Ovarian carcinoma is associated with the highest mortality of all gynecologic neoplasia worldwide, with a 5-year survival

Correspondence to: Dr Rouli Zhou, Department of Cell Biology, Peking University Health Science Center, Beijing 100083, P.R. China E-mail: rlzhou@bjmu.edu.cn

${ }^{*}$ Contributed equally

Key words: lysosome-associated protein transmembrane $4 \beta$, immunohistochemistry, ovarian carcinoma, survival rate of $<30 \%$ reported in some series $(1,2)$. The incidence of ovarian carcinoma has been steadily increasing over the past 10 years, with an overall lifetime risk of $1.8 \%$ (3). Despite advances in medical and surgical treatment and new chemotherapeutic regimens, the overall 5-year survival for women with epithelial ovarian carcinoma has remained almost unchanged over the past 40 years (4). The prognosis for patients with ovarian carcinoma can be determined by conventional evaluation using tumor histological subtype, histological grade, and International Federation of Gynecology and Obstetrics (FIGO) stage at time of diagnosis. However, these factors fail to fully take into account the spectrum of biological behavior of ovarian carcinoma, and more objective ways to establish prognosis are needed.

Recently, a novel gene lysosome-associated protein transmembrane $4 B$ (LAPTM4B) which was originally identified as a gene overexpressed in human hepatocellular carcinoma (HCC) was cloned by fluorescent differential-display polymerase chain reaction (PCR) (5). LAPTM4B, which maps to $8 \mathrm{q} 22$ and contains seven exons, encodes is a member of the mammalian 4-tetratransmembrane spanning protein superfamily. Analysis with immunohistochemical double labeling localized the protein predominantly to the intracellular membranes of lysosomes and late endosomes $(6,7)$. It has been shown that NIH3T3 cells transfected with LAPTM4B cDNA demonstrate higher proliferation rates, increased anchorage independent growth, reduced serum dependence, and changes in cell morphology (8). Moreover, the use of antisense oligonucleotides against LAPTM4B suppressed proliferation of the hepatocellular carcinoma cell line, BEL7402 (9). These results indicate that LAPTM4B plays an active role in cell proliferation during tumor development and/or progression rather than simply being upregulated as a secondary effect during tumorigenesis (10).

It was also recently reported that expression of LAPTM4B at mRNA and protein levels was highly upregulated in most HCCs and correlated inversely with HCC differentiation $(11,12)$. Current data have shown that LAPTM4B mRNA is also strongly expressed in several cancers, such as breast cancer, lung cancer, gastric cancer, colon cancer, uterus cancer, ovarian carcinoma, and adrenocorticotrophin (ACTH)-secreting and non-functioning 
pituitary adenoma $(10,12,13)$. However, there is no evidence concerning its protein expression status in ovarian carcinoma, and the clinicopathological relevance and significance of LAPTM4B overexpression in ovarian carcinoma remain unknown. The present study aimed to investigate expression of LAPTM4B in ovarian carcinoma and to evaluate its relationship to the clinicopathological features as noted in Table I, while evaluating its prognostic significance with univariate and multivariate analyses.

\section{Materials and methods}

Patients. After obtaining informed consent, 85 consecutive patients (ages 21-74 years, median age 52 years) with primary epithelial ovarian carcinoma served as subjects for this study. The patients underwent exploratory laparotomy at the Department of Obstetrics and Gynecology, Peking University Third Hospital (between 1998 and 2001). All tissue specimens underwent microscopic confirmation of diagnosis, tumor type, and histological grade by a pathologist specialized in gynaecological tumors. Histological typing was carried out according to the World Health Organisation (WHO) classification standards (14). The grading and staging of tumors were assigned according to the FIGO rules (15). The clinicopathological characteristics of the patients included in this study are summarized in Table I. Follow-up for the patients included in the survival analysis was completed by January 2008. Our research protocol was approved by Peking University Ethics Committee.

Immunoblot analysis. Seven frozen tissue samples of ovarian carcinoma and 2 fresh tissue samples of normal ovary were homogenised in a lysis buffer consisting of $1 \%$ Triton X-100 and a protease inhibitor mixture in phosphate-buffered saline. After centrifugation at $15,000 \mathrm{~g}$ for $20 \mathrm{~min}$, the supernatant was obtained. Thirty micrograms of protein extract were separated by SDS/10\% polyacrylamide gel electrophoresis, and transferred onto the nitrocellulose filters (Bio-Rad). The filters were blocked at $4{ }^{\circ} \mathrm{C}$ overnight with a blocking buffer (pH 7.6) containing 5\% non-fat dry milk. Filters were incubated with indicated antibody (1:300) for $2 \mathrm{~h}$ at room temperature, using rabbit anti-human polyclonal antibody (LAPTM4BN10-pAb), which specifically recognizes protein LAPTM4B35 (but not LAPTM4B-24). This antibody was raised in our laboratory, as previously reported (12). Immunoreactive proteins were stained using a chemiluminescence detection system (ECL, Amersham, Arlington Heights, IL, USA). Membranes were then washed with stripping solution for $1 \mathrm{~h}$ and treated as described above, except that mouse B-tubulin antibody (Neomarkers, Fremont, CA) was applied as an internal control.

LAPTM4B immunohistochemistry. LAPTM4B expression was evaluated in 85 patients with ovarian carcinoma with immunohistochemistry using paraffin-embedded specimens. Surgical specimens were fixed in $10 \%$ formalin, embedded in paraffin, and were sectioned at a thickness of $4 \mu \mathrm{m}$. For heatinduced epitope retrieval, deparaffinised sections were soaked in $10 \mathrm{mM}$ citrate buffer ( $\mathrm{pH} 6.0$ ) and treated at $95^{\circ} \mathrm{C}$ for $30 \mathrm{~min}$ in a microwave oven.

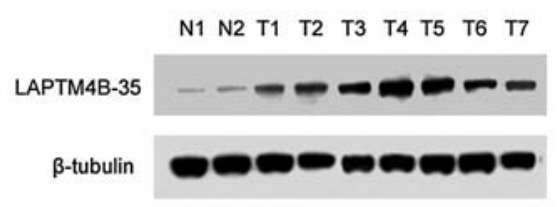

Figure 1. LAPTM4B expression in normal ovaries and ovarian carcinoma tissues. Lane N1, N2: 2 fresh normal ovaries. Lane T1-T7: 7 frozen ovarian carcinomas. B-tubulin expression is shown to demonstrate equal protein loading.

Immunohistochemical staining was performed using the avidin-biotin immunoperoxidase technique. Endogenous peroxidase activity was first blocked by incubation with $0.3 \%$ $\mathrm{H}_{2} \mathrm{O}_{2}$ in methanol for $15 \mathrm{~min}$, and non-specific immunoglobulin binding was then blocked by incubation with $10 \%$ normal goat serum for $10 \mathrm{~min}$. Sections were incubated at room temperature for $2 \mathrm{~h}$ with the anti-LAPTM4B polyclonal antibody at 1:100 dilution. The sections were rinsed and incubated for $30 \mathrm{~min}$ with biotinylated second antibody. After washing, the sections were incubated for $30 \mathrm{~min}$ with horseradish peroxidase-conjugated streptavidin, and finally treated with 3,3'-diaminobenzidine tetrahydrochloride in $0.01 \% \mathrm{H}_{2} \mathrm{O}_{2}$ for $10 \mathrm{~min}$. The slides were counterstained with Meyer's hematoxylin. As a negative control, the primary antibody was replaced with normal rabbit IgG at an appropriate dilution.

Evaluation of the stainings. The LAPTM4B expression levels were classified semiquantitatively based on the total combined scores of the percent positive staining tumor cells together with the staining intensity. A tumor was scored as ' 0 ' if $<10 \%$ of tumor cells stained positive, ' 1 ' if $10-50 \%$ were positive, and ' 2 ' if $>50 \%$ of cells were positive stained. The staining intensity was scored as ' 0 ' when no cells stained or there was only weak staining, ' 1 ' when there was moderate staining, and ' 2 ' in cases of strong staining. The final LAPTM4B expression score was defined as 'Low LAPTM4B expression' when the sum of the positivity score and the staining intensity score was $0-2$, and 'High LAPTM4B expression' when the sum was 3-4. In each case, at least 3 different areas of tumor were evaluated, and the mean of the results was taken as the final expression score. The scoring procedure was carried out twice by two independent observers without any knowledge of the clinical data and without review of corresponding H\&E slides. The concordance rate between the two primary observers was $>95 \%$. In cases of significant disagreement, the slides in question were re-reviewed simultaneously by the original two observers, together with a third observer together at a multiheaded microscope, in order to resolve the difference of opinion.

Statistical analyses. The Chi-square test was used to analyze the correlation of LAPTM4B expression with various clinicopathological parameters. Overall survival (OS) was calculated as the interval from the date of surgery to the date of death, and progression-free survival (PFS) was the interval from the date of surgery to the date of progression/recurrence, or date of last follow-up examination. Univariate analysis for 
Table I. LAPTM4B expression in relation to age, FIGO stage, histological subtype, histological grade, and residual tumor in ovarian carcinomas.

\begin{tabular}{|c|c|c|c|c|}
\hline \multirow[b]{2}{*}{ Variables } & \multirow[b]{2}{*}{ Patients } & \multicolumn{2}{|c|}{ LAPTM4B expression } & \multirow[b]{2}{*}{$\mathrm{P}^{\mathrm{a}}$} \\
\hline & & Low & High & \\
\hline All cases & 85 & 31 & 54 & \\
\hline Age (years) & & & & 0.73 \\
\hline$>60$ & 21 & 7 & 14 & \\
\hline$\leq 60$ & 64 & 24 & 40 & \\
\hline FIGO stage & & & & 0.03 \\
\hline I & 20 & 11 & 9 & \\
\hline II & 12 & 7 & 5 & \\
\hline III & 45 & 11 & 34 & \\
\hline IV & 8 & 2 & 6 & \\
\hline Histological subtype & & & & 0.02 \\
\hline Serous & 43 & 8 & 35 & \\
\hline Mucinous & 15 & 10 & 5 & \\
\hline Endometrioid & 10 & 4 & 6 & \\
\hline Clear cell & 9 & 4 & 5 & \\
\hline Other type & 8 & 5 & 3 & \\
\hline Histological grade & & & & 0.39 \\
\hline 1 & 13 & 7 & 6 & \\
\hline 2 & 33 & 11 & 22 & \\
\hline 3 & 39 & 13 & 26 & \\
\hline Residual tumor & & & & $<0.001$ \\
\hline Negative & 38 & 23 & 15 & \\
\hline Positive & 47 & 8 & 39 & \\
\hline
\end{tabular}

FIGO, International Federation of Gynecology and Obstetrics; LAPTM4B, lysosome-associated protein transmembrane-4 B and ${ }^{\mathrm{a} C h i}$-square test.

OS and PFS was performed and evaluated with the log-rank test, and Kaplan-Meier curves were generated. A multivariate Cox proportional-hazard model was used to evaluate the independence of LAPTM4B expression as prognostic factor against other variables such as age, FIGO stage, histological subtype and residual tumor at laparotomy. For this statistical evaluation SPSS software Version 10.0 was used, and a P-value $\leq 0.05$ was considered significant.

\section{Results}

Clinical profiles of the patients. Of 85 patients, $20(23.5 \%)$ were FIGO stage I, 12 (14.1\%) were stage II, 45 (52.9\%) were stage III, and $8(9.4 \%)$ were stage IV. Histological subtypes of the tumor included $43(50.6 \%)$ cases of serous ovarian carcinoma, $15(17.6 \%)$ cases of mucinous carcinoma, 10 $(11.8 \%)$ cases of endometrioid carcinoma, $9(10.6 \%)$ cases of clear cell carcinoma, and $8(9.4 \%)$ cases of other types (Table I). At the end of the follow-up period, 28 (32.9\%) patients were alive, $53(62.4 \%)$ patients had died of disease, and $4(4.7 \%)$ of the patients were lost to follow-up. The median follow-up was 53 months (range 4-119).

LAPTM4B protein expression in ovarian carcinoma tissues by Western blot analysis. Western blot analysis was performed to investigate LAPTM4B protein expression in 7 frozen ovarian carcinomas and 2 fresh normal ovaries. In all samples, LAPTM4B protein was detected as $\sim 35 \mathrm{kDa}$ bands (Fig. 1). Although LAPTM4B protein was expressed constitutively in normal ovary, its expression was lower than that in ovarian carcinoma. Increased LAPTM4B protein expression was observed in ovarian carcinomas, although its expression level varied among the samples. This observation was consistent with the different percentage of positive stained tumor cells and varying staining intensity among ovarian carcinomas identified by immunohistochemical analysis.

Expression of LAPTM4B and its relationship with clinicopathological features of ovarian carcinoma. As shown in Fig. 2A-F, the immunoreactivity of LAPTM4B was detected 
Table II. Univariate survival analysis of OS and PFS in 81 patients with ovarian carcinoma.

\begin{tabular}{|c|c|c|c|c|c|c|c|}
\hline \multirow[b]{2}{*}{ Variables } & \multirow[b]{2}{*}{$\begin{array}{l}\text { No. of } \\
\text { cases }\end{array}$} & \multicolumn{3}{|c|}{ OS } & \multicolumn{3}{|c|}{ PFS } \\
\hline & & $\begin{array}{c}\text { Mean } \pm \text { SE } \\
\text { (month) }\end{array}$ & $\begin{array}{l}\text { Median } \pm \mathrm{SE} \\
\quad \text { (month) }\end{array}$ & $\mathrm{P}^{\mathrm{a}}$ & $\begin{array}{c}\text { Mean } \pm \text { SE } \\
\text { (month) }\end{array}$ & $\begin{array}{l}\text { Median } \pm \mathrm{SE} \\
\quad \text { (month) }\end{array}$ & $\mathrm{P}^{\mathrm{a}}$ \\
\hline FIGO stage & & & & $<0.001$ & & & $<0.001$ \\
\hline I & 19 & $104 \pm 7$ & NR & & $96 \pm 9$ & NR & \\
\hline II & 11 & $87 \pm 10$ & NR & & $71 \pm 12$ & $56 \pm 0$ & \\
\hline III & 43 & $58 \pm 5$ & $50 \pm 3$ & & $31 \pm 4$ & $25 \pm 2$ & \\
\hline IV & 8 & $16 \pm 3$ & $13 \pm 3$ & & $8 \pm 2$ & $6 \pm 3$ & \\
\hline Age (years) & & & & 0.012 & & & 0.032 \\
\hline$>60$ & 20 & $49 \pm 8$ & $55 \pm 6$ & & $31 \pm 7$ & $23 \pm 7$ & \\
\hline$\leq 60$ & 61 & $77 \pm 5$ & $70 \pm 13$ & & $56 \pm 6$ & $34 \pm 6$ & \\
\hline Histological grade & & & & 0.003 & & & 0.001 \\
\hline 1 & 12 & $102 \pm 9$ & NR & & $92 \pm 11$ & NR & \\
\hline 2 & 32 & $74 \pm 7$ & $70 \pm 16$ & & $53 \pm 8$ & $31 \pm 8$ & \\
\hline 3 & 37 & $54 \pm 6$ & $48 \pm 5$ & & $32 \pm 5$ & $21 \pm 3$ & \\
\hline Histological subtype & & & & 0.045 & & & 0.007 \\
\hline Serous & 43 & $57 \pm 6$ & $50 \pm 5$ & & $36 \pm 6$ & $21 \pm 2$ & \\
\hline Mucinous & 15 & $88 \pm 9$ & NR & & $76 \pm 11$ & NR & \\
\hline Endometrioid & 9 & $83 \pm 11$ & NR & & $64 \pm 14$ & $38 \pm 16$ & \\
\hline Clear cell & 8 & $66 \pm 8$ & $70 \pm 18$ & & $41 \pm 7$ & $38 \pm 8$ & \\
\hline Other type & 6 & $86 \pm 12$ & $59 \pm 0$ & & $71 \pm 18$ & $32 \pm 0$ & \\
\hline Residual tumor & & & & $<0.001$ & & & $<0.001$ \\
\hline+ & 46 & $43 \pm 3$ & $46 \pm 2$ & & $21 \pm 2$ & $21 \pm 2$ & \\
\hline- & 35 & $101 \pm 5$ & NR & & $90 \pm 7$ & NR & \\
\hline LAPTM4B expression & & & & $<0.001$ & & & $<0.001$ \\
\hline Low & 28 & $100 \pm 7$ & NR & & $85 \pm 8$ & NR & \\
\hline High & 53 & $53 \pm 4$ & $48 \pm 2$ & & $32 \pm 4$ & $23 \pm 2$ & \\
\hline
\end{tabular}

OS, overall survival; PFS, progression-free survival; LAPTM4B, lysosome-associated protein transmembrane-4 bß; NR, not reached and ${ }^{a} \log$-rank test.

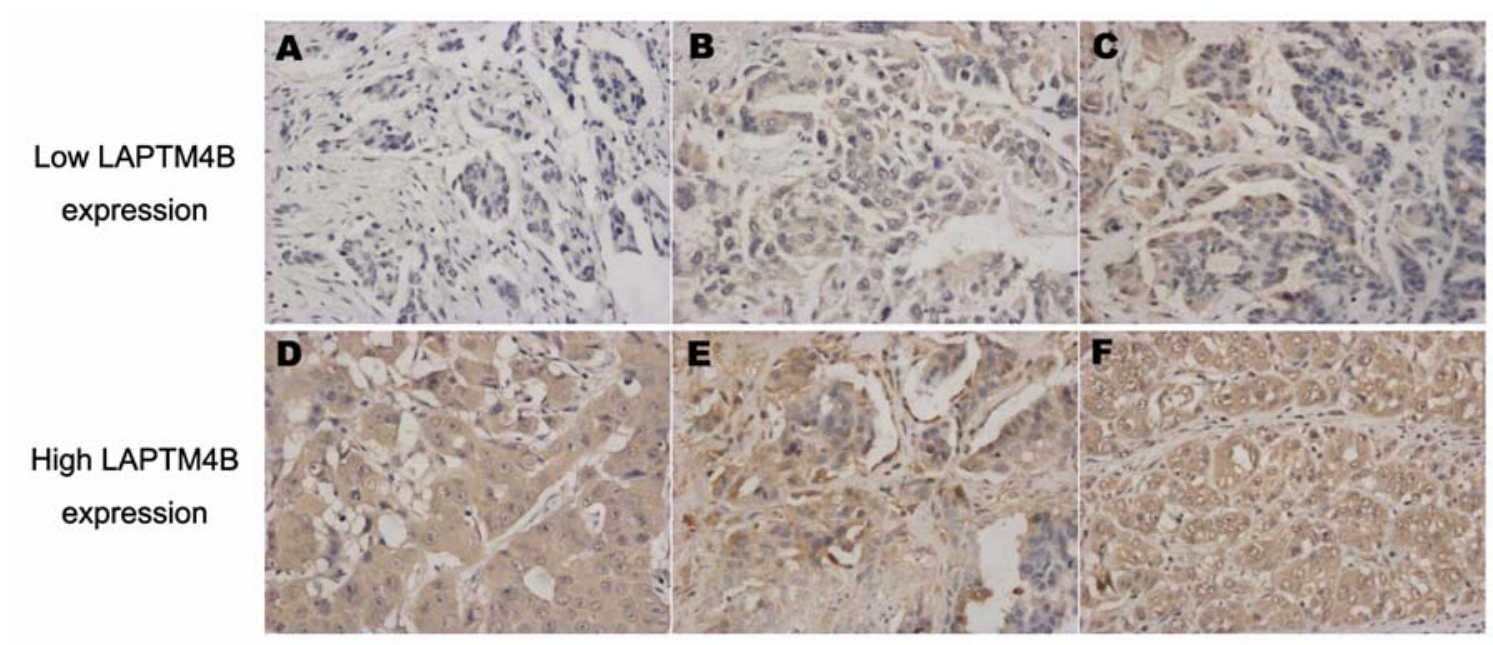

Figure 2. (A-C) Representative staining pattern of low LAPTM4B expression (positivity+intensity). A, score of 0+0; B, score of 1+0 and C, score of 1+1. (D-F) Representative staining pattern of low LAPTM4B expression (positivity + intensity). D, score of $2+1$; E, score of $1+2$ and F, score of $2+2$. Original magnification, x200. 
Table III. Multivariate survival analysis of OS and PFS in 81 patients with ovarian carcinoma.

\begin{tabular}{lcccccc}
\hline & \multicolumn{3}{c}{ OS } & & \multicolumn{2}{c}{ PFS } \\
\cline { 2 - 3 } Variables & RR & $95 \%$ CI & P & RR & $95 \%$ CI & P \\
\hline FIGO stage & 3.19 & $1.42-7.2$ & 0.005 & 2.58 & $1.26-5.27$ & 0.009 \\
Age (year) & 0.91 & $0.45-1.87$ & 0.800 & 1.03 & $0.54-1.97$ & 0.940 \\
Histological grade & 1.15 & $0.64-2.07$ & 0.640 & 1.14 & $0.66-1.99$ & 0.640 \\
Histological subtype & 0.73 & $0.57-0.93$ & 0.010 & 0.72 & $0.57-0.90$ & 0.004 \\
Residual tumor & 1.38 & $0.45-4.22$ & 0.570 & 2.67 & $0.89-8.00$ & 0.080 \\
LAPTM4B expression & 3.37 & $1.33-8.52$ & 0.010 & 2.33 & $1.07-5.09$ & 0.030 \\
\hline
\end{tabular}

OS, overall survival; PFS, progression-free survival; RR, relative risk; CI, confidence interval; LAPTM4B, lysosome-associated protein transmembrane- $4 \beta$ and ${ }^{\text {a }}$ Cox regression test.

at variable levels, and was localised in the cytoplasm of tumor cells. Of the 85 specimens examined, LAPTM4B expression was low in 31 of 85 patients $(36.5 \%)$ and high in 54 of 85 patients $(63.5 \%)$ as defined by the immunohistochemical criteria above. Table I summarizes the relationship between LAPTM4B expression and clinicopathological features in ovarian carcinomas. LAPTM4B expression showed correlation with FIGO stage $(\mathrm{P}=0.03)$, histological subtype $(\mathrm{P}=0.02)$ and residual tumor after primary surgery $(\mathrm{P}<0.01)$. However, no significant association was found between LAPTM4B expression and age and histological grade.

The impact of LAPTM4B expression on overall and progression-free survival in ovarian carcinomas. The Kaplan-Meier curve and log-rank test showed that the 5-year survival rate of patients with high expression of LAPTM4B was significantly worse than that with low expression (OS rate $\pm \mathrm{SD}$, low expression vs. high: $82 \pm 7 \%$ vs. $21 \pm 6 \%$, $\mathrm{P}<0.001$; PFS rate \pm SD, low expression vs. high: $61 \pm 9 \%$ vs. $11 \pm 4 \%, \mathrm{P}<0.001$ ) (Fig. 3). The OS period (mean months $\pm \mathrm{SE}$ ) of the LAPTM4B low vs. high expression group was $100 \pm 7$ vs. $53 \pm 4(\mathrm{P}<0.001)$, and the PFS period (mean months $\pm \mathrm{SE})$ was $85 \pm 8$ vs. $32 \pm 4(\mathrm{P}<0.001)$ (Table II).

To determine whether LAPTM4B expression is independent of FIGO stage as a prognostic factor, we calculated OS and PFS analysis with the use of Univariate analysis and the Cox proportional hazards model. Univariate analysis revealed that FIGO stage, age, histological grade, histological subtype, residual tumor, and LAPTM4B expression significantly affected on the survival of the patients with ovarian carcinomas (Table II). Therefore, we performed multivariate analysis with FIGO stage, age, histological grade, histological subtype, residual tumor, and LAPTM4B expression, and the results are shown in Table III. These findings indicate that in this study LAPTM4B expression was an independent prognostic factor (Table III, RR, 3.37; 95\% CI, 1.33-8.52; $\mathrm{P}=0.01$ and $\mathrm{RR}, 2.33 ; 95 \% \mathrm{CI}, 1.07-5.09$; $\mathrm{P}=0.03$ ) for $\mathrm{OS}$ and PFS of ovarian carcinoma patients. FIGO stage was another factor which was also significantly independently associated with OS and PFS (RR, 3.19; 95\% CI, 1.41-7.20; $\mathrm{P}=0.005$ and $\mathrm{RR}, 2.58 ; 95 \% \mathrm{CI}, 1.26-5.27$; $\mathrm{P}=0.009)$. Histological subtype was another independent

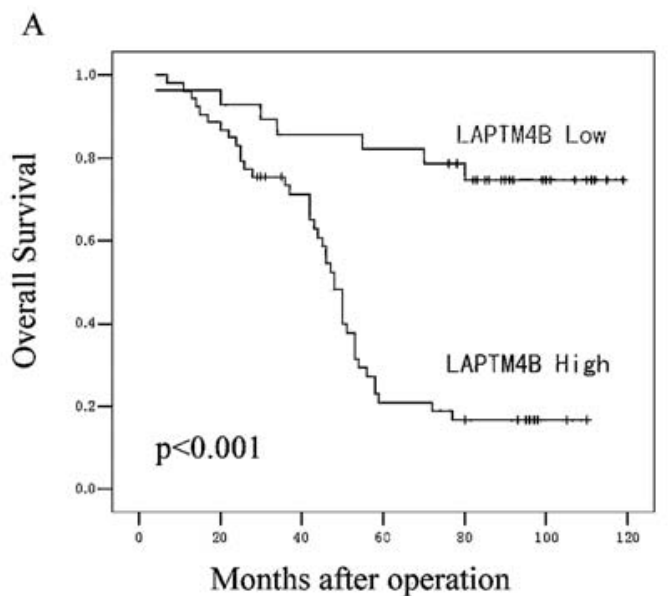

B

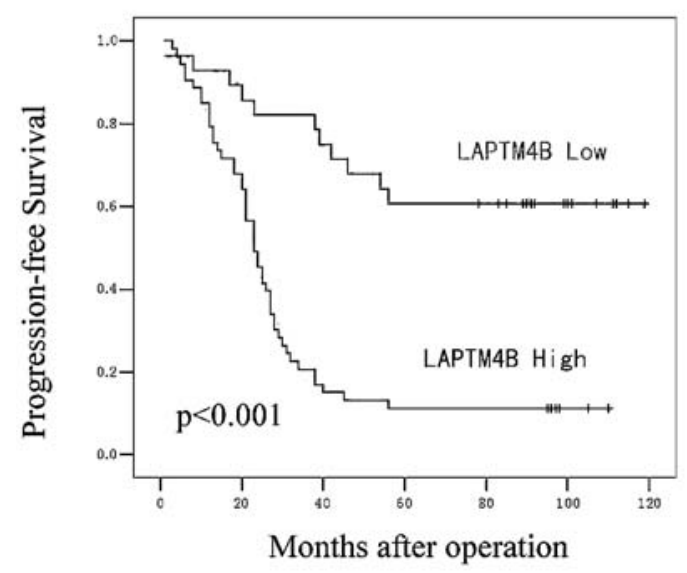

Figure 3. (A) Kaplan-Meier curves for overall survival of ovarian carcinoma patients according to the percentage of low and high expression of LAPTM4B (log-rank analysis). (B) Kaplan-Meier progression-free survival curves of ovarian carcinoma patients according to the percentage of low and high expression of LAPTM4B (log-rank analysis).

prognostic factor $(\mathrm{RR}, 0.73 ; 95 \% \mathrm{CI}, 0.57-0.93 ; \mathrm{P}=0.01$ and RR, 0.72; 95\% CI, 0.57-0.9; P=0.004) for OS and PFS of ovarian carcinoma patients. 


\section{Discussion}

In this study we analyzed LAPTM4B expression by immunohistochemistry in ovarian carcinoma using 85 surgical specimens, and found that LAPTM4B was an independent prognostic factor, and high expression of LAPTM4B by tumor cells was associated with poor survival. This is the first study to demonstrate in detail an association of selected clinicopathological features and prognostic impact of LAPTM4B expression in ovarian carcinoma using a large number of clinical samples.

We analyzed the correlation of LAPTM4B expression with traditional clinicopathological parameters in ovarian carcinoma. High LAPTM4B expression was significantly correlated with histological subtype, advanced tumor stage and the presence of residual tumor after primary surgery, but may not with the age and histological grade of ovarian carcinomas. These results suggest that the high LAPTM4B expression is associated with the disease progression of ovarian carcinoma, and raises the possibility that its expression reflects tumor aggressiveness rather than the grade of tumor differentiation.

The present data demonstrated clearly that the patients with high LAPTM4B expression had an unfavorable clinical outcome by analysing the rates of OS and DFS. In this study, multivariate analysis demonstrated that among the factors analyzed, with the possible exception of the FIGO stage and histological subtype, LAPTM4B expression was an independent prognostic factor for OS and PFS in ovarian carcinoma patients. This raises the possibility that LAPTM4B may be a prognostic parameter for ovarian carcinoma which is as or more reliable than the clinicopathological factors currently in use, and suggests the possibility of use of LAPTM4B in individualization of adjuvant therapy wherein patients who have high LAPTM4B expression could be given more aggressive adjuvant therapy based on higher likelihood of unfavorable biological behavior. It is of note that several other molecular markers such as COX-2, Ep-CAM, Skp2 and survivin have been identified which show association with prognosis in ovarian carcinoma (16-19). However, it is not yet clear whether such markers may be most effective for clinical application as replacements for, or in addition to, the prognostic factors currently in use. As such, further investigation is called for to determine whether combined detection of LAPTM4B together with some of these other molecules would be of value in enhancing prognostic effectiveness.

This study is consistent with the previously reported results with LAPTM4B in HCC (12), and is also consistent with the recent study of Zhou et al (20) which showed correlation between LAPTM4B expression and prognosis in gallbladder carcinoma suggesting that patients with high LAPTM4B expression are very likely to recur and have a poor prognosis. In a similar manner, our data support a relationship between high expression of LAPTM4B protein and unfavorable biological behavior in ovarian carcinoma. This raises the possibility that the LAPTM4B gene may play a pivotal role in progression of some solid malignancies such as ovarian carcinoma, HCC and BGC. However, the mechanism by which LAPTM4B expression contributes to tumor progression of ovarian carcinoma remains to be worked out. A recent report proposed a mechanism whereby upregulation of LAPTM4B in tumors of organs which are sex hormone dependent (e.g. tumors of the ovary, breast and uterus) could interfere with intracellular steroid distribution and hence decrease their availability to corresponding receptors, leading to the uncontrolled tumor progression by promoting differentiation and proliferation (21). Thus, the correlation of the high LAPTM4B expression with disease progression and the impaired patient survival shown in the present study may be attributable to dysregulation of intracellular steroid by the tumor-mediated LAPTM4B activity. However, further investigation including evaluation of hormone receptor status in these patients is needed to specifically identify the pathobiologic roles of LAPTM4B in cancer cells.

It was also found that the sequences of 91 amino acids at the N-terminus of LAPTM4B were essential for its functions on cell survival and growth (9). The N-terminal stretch of LAPTM4B contains a PXXP motif flanked by hydrophobic residues. As this is characteristic for ligands of src-homology3 domains (SH3) such as PI3K, PP2A and PKC, LAPTM4B is likely to interact with further downstream target proteins (5). Furthermore, the N-terminus of LAPTM4B contains several potential phosphorylation sites for $\mathrm{cAMP} / \mathrm{cGMP}$-dependent protein kinases as well as for protein kinase C (5). Consistently, coimmunoprecipitation assay indicated that LAPTM4B interacted with integrin- $\alpha 631$ in BEL-7402 cells, which were enhanced by LN-1(22), and may play an important role in the integrin- $\alpha 6$ mediating signal transduction pathways. These data indicate an involvement of LAPTM4B in signal transduction pathway(s) thus enhancing cell proliferation or survival. However, the role of LAPTM4B in signal transduction pathways in tumor cells is certainly worth further intense investigation.

In conclusion, we demonstrated here that LAPTM4B is commonly expressed in ovarian carcinoma and that high LAPTM4B expression as determined by immunohistochemistry correlated with the disease progression and poor clinical outcome in ovarian carcinoma patients. Furthermore, LAPTM4B proved to be an independent molecular marker of prognosis in ovarian carcinoma in this study and may become a novel molecular target in the strategies for clinical evaluation and treatment of this disease.

\section{References}

1. Cannistra SA: Cancer of the ovary. N Engl J Med 351: 2519-2529, 2004.

2. Seidman JD and Kurman RJ: Pathology of ovarian carcinoma. Hematol Oncol Clin North Am 17: 909-925, 2003.

3. Jemal A, Thomas A, Murray T and Thun M: Cancer statistics. CA Cancer J Clin 52: 23-47, 2002.

4. Ozols R, Rubin SC, Thomas GM and Robboy SJ: Principles and Practice of Gynecologic Oncology. 3rd edition, Lippincott Williams \& Wilkins, Philadelphia, 2000.

5. Shao GZ, Zhou RL, Zhang QY, Zhang Y, Liu JJ, Rui JA, Wei X and Ye DX: Molecular cloning and characterization of LAPTM4B, a novel gene upregulated in hepatocellular carcinoma. Oncogene 22: 5060-5069, 2003.

6. Hogue DL, Nash C, Ling V and Hobman TC: Lysosome-associated protein transmembrane 4 alpha (LAPTM4 alpha) requires two tandemly arranged tyrosine-based signals for sorting to lysosomes. Biochem J 365: 721-730, 2002.

7. Hunziker W and Geuze HJ: Intracellular trafficking of lysosomal membrane proteins. Bioessays 18: 379-389, 1996. 
8. He J, Shao G and Zhou R: Effects of the novel gene, LAPTM4B, highly expression in hepatocellular carcinoma on cell proliferation and tumorigenesis of NIH3T3 cells. Beijing Da Xue Xue Bao 35: 348-352, 2003.

9. Liu J, Zhou R, Zhang N, Rui J and Jin C: Biological function of a novel gene overexpressed in human hepatocellular carcinoma. Chin Med J 113: 881-885, 2000.

10. Kasper G, Vogel A, Klaman I, Grone J, Petersen I, Weber B, Castanos-Velez E, Staub E and Mennerich D: The human LAPTM4b transcript is upregulated in various types of solid tumours and seems to play a dual functional role during tumour progression. Cancer Lett 224: 93-103, 2005.

11. Liu XR, Zhou RL, Zhang QY, Zhang Y, Jin YY, Lin M, Rui JA and Ye DX: Structure analysis and expressions of a novel tetratransmembrane protein, lysosoma-associated protein transmembrane $4 \beta$ associated with hepatocellular carcinoma. World J Gastroenterol 10: 1555-1559, 2004.

12. Peng C, Zhou RL, Shao GZ, Rui JA, Wang SB, Lin M, Zhang S and Gao ZF: Expression of lysosome-associated protein transmembrane 4B-35 in cancer and its correlation with the differentiation status of hepatocellular carcinoma. World J Gastroenterol 11: 2704-2708, 2005

13. Morris DG, Musat M, Czirjak S, Hanzely Z, Lillington DM, Korbonits $\mathrm{M}$ and Grossman AB: Differential gene expression in pituitary adenomas by oligonucleotide array analysis. Eur J Endocrinol 153: 143-151, 2005.

14. Scully R: Histological typing of ovarian tumors. In: WHO International Classification of Tumours. Springer-Verlag, Berlin, 1999.

15. Benedet JL, Bender H, Jones H, Ngan HY and Pecorelli S: FIGO staging classifications and clinical practice guidelines in the management of gynecologic cancers. FIGO Committee on Gynecologic Oncology. Int J Gynaecol Obstet 70: 209-262, 2000.
16. Denkert C, Kobel M, Pest S, Koch I, Berger S, Schwabe M, Siegert A, Reles A, Klosterhalfen B and Hauptmann S: Expression of cyclooxygenase 2 is an independent prognostic factor in human ovarian carcinoma. Am J Pathol 160: 893-903, 2002.

17. Spizzo G, Went P, Dirnhofer S, Obrist P, Simon R, Spichtin H, Maurer R, Metzger U, von Castelberg B, Bart R, Stopatschinskaya S, Kochli OR, Haas P, Mross F, Zuber M, Dietrich H, Bischoff S, Mirlacher M, Sauter G and Gastl G: High Ep-CAM expression is associated with poor prognosis in node-positive breast cancer. Breast Cancer Res Treat 86: 207-213, 2004.

18. Shigemasa K, Gu L, O'Brien TJ and Ohama K: Skp2 overexpression is a prognostic factor in patients with ovarian adenocarcinoma. Clin Cancer Res 9: 1756-1763, 2003.

19. Sui L, Dong Y, Ohno M, Watanabe Y, Sugimoto K and Tokuda M: Survivin expression and its correlation with cell proliferation and prognosis in epithelial ovarian tumors. Int J Oncol 21: 315320,2002

20. Zhou L, He XD, Chen J, Cui QC, Qu Q, Rui JA and Zhao YP. Overexpression of LAPTM4B-35 closely correlated with clinicopathological features and post-resectional survival of gallbladder carcinoma. Eur J Cancer 43: 809-815, 2007.

21. Hogue DL, Kerby L and Ling V: A mammalian lysosomal membrane protein confers multidrug resistance upon expression in Saccharomyces cerevisiae. J Biol Chem 274: 12877-12882, 1999.

22. Liu X, Zhou R, Zhang Q, Zhang Y, Shao G, Jin Y, Zhang S, Lin M, Rui J and Ye D: Identification and characterization of LAPTM4B encoded by a human hepatocellular carcinoma-associated novel gene. Beijing Da Xue Xue Bao 35: 340-347, 2003. 